ARTICLE

\title{
Spectral Analysis of Stochastic Noise in Fission Source Distributions from Monte Carlo Eigenvalue Calculations
}

\author{
David P. GRIESHEIMER* and Brian R. NEASE \\ Bettis Atomic Power Laboratory, Bechtel Marine Propulsion Corporation, West Mifflin, PA, 15122, USA
}

\begin{abstract}
In this paper a new analysis of the characteristics and propagation of random fluctuations in Monte Carlo (MC) eigenvalue calculations is presented. In particular, random fluctuations in the fission source distribution produced from a fixed set of initial neutron source locations are considered. Unlike previous analyses, this work relies on spectral theory to analyze a general class of MC eigenvalue transport algorithms. This new analysis uses a simple delta function representation to express the set of discrete neutron source locations as a continuous function, which can be written in terms of the eigenfunctions of the transport operator. This allows the iterative MC source convergence process to be analyzed using existing techniques developed for the traditional power iteration method. Furthermore, it can be shown that the random fluctuations introduced during these source iterations are due to the fact that MC algorithms must approximate the continuous fission source distribution by a discrete set of randomly sampled fission sites. Based on this result, a series expansion for the MC fission source realization is derived, and the expected value of the expansion coefficients for this series are shown to converge to the expansion coefficients for the continuous fission source distribution. An expression for the variance of each of the MC fission distribution expansion coefficients is also presented. Numerical results for a simple 1-D slab geometry problem are presented to support the theoretical results derived in the paper.
\end{abstract}

KEYWORDS: Monte Carlo, noise analysis, spectral theory, source convergence, power method

\section{Introduction}

One of the unique challenges presented by Monte Carlo (MC) particle transport methods is the presence of statistical uncertainty in the solutions. These statistical uncertainties are due to the stochastic nature of the underlying MC process itself. Each solution produced by a fair-game MC algorithm is a single realization of the transport process, based on a finite number of independent neutron histories. Due to the finite sample size, each MC realization will show differences when compared to the true (expected) solution. The differences between the realized and expected solutions appear as random fluctuations. Unfortunately, the magnitude of these fluctuations cannot simply be estimated by the sample variance in eigenvalue transport problems because the iterative process used to converge the solution causes fluctuations introduced during one iteration to propagate to all subsequent iterations. As a result, the statistical uncertainty of the final solution is more difficult to compute because it depends on the shared uncertainties of all realizations.

The convergence of eigenmodes during $\mathrm{MC}$ calculations has been considered in several previous studies. ${ }^{1-4)}$ In particular, the random fluctuations of the individual eigenmodes during MC source iterations are especially relevant when considering extrapolation methods for source convergence acceleration. In his 2009 paper, Toth points out that source extrapolation techniques tend to amplify both the source convergence behavior as well as any random noise compo-

*Corresponding author, E-mail: dgrieshe@umich.edu

(c) 2011 Atomic Energy Society of Japan, All Rights Reserved. nents that may be present in the source distribution. ${ }^{4)}$ Thus, as the source approaches convergence extrapolation methods become counter-productive as they begin to simply increase the random noise present in the source distribution. This observation has led Toth to propose the existence of a break-even point during source convergence beyond which source extrapolation methods should not be used. In an effort to identify this break-even point, Toth proposed that the fluctuations on each of the source eigenmodes could be characterized by a single, zero-mean, random variable. ${ }^{4)}$ In addition, during the course of his analysis, Toth also made three assumptions regarding the characteristics of the eigenmode noise terms: 1) the random variables are normally distributed with zero-mean, 2) the variance of the noise terms are constant for all eigenmodes, and 3) the noise terms are independent between eigenmodes. These assumptions were based on experimental evidence for a limited set of problems of interest, and showed good agreement with experimental results. ${ }^{4)}$

This paper attempts to provide a more rigorous theoretical foundation for describing the random fluctuations affecting individual eigenmodes during MC source iterations. Understanding the fluctuations for each eigenmode is an important step in achieving a better understanding of the MC source convergence process and possibly developing improved source acceleration and convergence detection algorithms. In addition, we will consider the reasonability of Toth's assumptions regarding the statistical properties of the eigenmode fluctuations. 


\section{Theory}

\section{Derivation of Neutron Transport Operator}

To begin, let us consider a three-dimensional multiplying material with volume $V$. The macroscopic production and absorption cross sections throughout the volume are given by the functions $v \Sigma_{f}(\vec{r}, E)$ and $\Sigma_{a}(\vec{r}, E)$, respectively. For completeness we seek to derive the general neutron transport eigenvalue problem, which is to be solved by Monte Carlo simulation. The derivation is similar to an early approach originally outlined by Goad and Johnson in their 1959 paper on Monte Carlo criticality methods. ${ }^{5)}$ We start with the steady-state, fixed source neutron transport equation

$$
\begin{aligned}
& \hat{\Omega} \cdot \nabla \psi(\vec{r}, E, \hat{\Omega})+\Sigma_{t}(\vec{r}, E) \psi(\vec{r}, E, \hat{\Omega}) \\
& \quad-\int_{0}^{\infty} \int_{4 \pi} \Sigma_{s}\left(\vec{r}, E^{\prime} \rightarrow E, \hat{\Omega}^{\prime} \rightarrow \hat{\Omega}\right) \psi\left(\vec{r}, E^{\prime}, \hat{\Omega}^{\prime}\right) d \hat{\Omega}^{\prime} d E^{\prime}=Q(\vec{r}, E, \hat{\Omega}) .
\end{aligned}
$$

The notation adopted in Eq. (1) is standard within the nuclear engineering community, where $\psi(\vec{r}, E, \hat{\Omega})$ and $Q(\vec{r}, E, \hat{\Omega})$ represent the angular neutron flux and fixed neutron source density, respectively, per unit volume, energy, and solid angle. For simplicity we will drop the energy dependence of Eq. (1) and consider only the one speed problem, which is governed by

$$
\begin{aligned}
& \hat{\Omega} \cdot \nabla \psi(\vec{r}, \hat{\Omega})+\Sigma_{t}(\vec{r}) \psi(\vec{r}, \hat{\Omega}) \\
& \quad-\int_{4 \pi} \Sigma_{s}\left(\vec{r}, \hat{\Omega}^{\prime} \rightarrow \hat{\Omega}\right) \psi\left(\vec{r}, \hat{\Omega}^{\prime}\right) d \hat{\Omega}^{\prime}=Q(\vec{r}, \hat{\Omega}) .
\end{aligned}
$$

Strictly speaking, the one-speed approximation is not required for the following derivations to hold. However, if the one-speed approximation is not used then the general solution will require eigenfunction solutions of multiple independent variables (position and energy) and is more cumbersome to work with.

Given Eq. (2), the flux due to a single point source at $\vec{r}_{0}, \hat{\Omega}_{0}$ can be written in terms of a general Green's function, $G$, such that

$$
\psi(\vec{r}, \hat{\Omega})=G\left(\vec{r}_{0} \rightarrow \vec{r}, \hat{\Omega}_{0} \rightarrow \hat{\Omega}\right) Q\left(\vec{r}_{0}, \hat{\Omega}_{0}\right) .
$$

By superposition it holds that the flux for an isotropic neutron source $S(\vec{r})$ arbitrarily distributed in volume $V$ is given by

$$
\psi(\vec{r}, \hat{\Omega})=\frac{1}{4 \pi} \int_{V} \int_{4 \pi} G\left(\vec{r}^{\prime} \rightarrow \vec{r}, \hat{\Omega}^{\prime} \rightarrow \hat{\Omega}\right) S\left(\vec{r}^{\prime}\right) d \hat{\Omega}^{\prime} d \vec{r}^{\prime} .
$$

Again, the assumption of an isotropic neutron source is not strictly necessary but it is appropriate for neutron sources due to fission and will simplify the following derivations.

From Eq. (4) we can immediately compute the total absorption rate density for any point $\vec{r}$ in the system

$$
\begin{aligned}
& \sum_{a}(\vec{r}) \int_{4 \pi} \psi(\vec{r}, \hat{\Omega}) d \hat{\Omega} \\
& \quad=\frac{\sum_{a}(\vec{r})}{4 \pi} \int_{V} \int_{4 \pi} \int_{4 \pi} G\left(\vec{r}^{\prime} \rightarrow \vec{r}, \hat{\Omega}^{\prime} \rightarrow \hat{\Omega}\right) S\left(\vec{r}^{\prime}\right) d \hat{\Omega} d \hat{\Omega}^{\prime} d \vec{r}^{\prime} .
\end{aligned}
$$

Let us now define the right hand side of Eq. (5) as the neutron absorption operator $\mathbf{A}$

$$
\mathbf{A}[S(\vec{r})] \equiv \frac{\sum_{a}(\vec{r})}{4 \pi} \int_{V} \int_{4 \pi} \int_{4 \pi} G\left(\vec{r}^{\prime} \rightarrow \vec{r}, \hat{\Omega}^{\prime} \rightarrow \hat{\Omega}\right) S\left(\vec{r}^{\prime}\right) d \hat{\Omega} d \hat{\Omega}^{\prime} d \vec{r}^{\prime} .
$$

The operator A gives the rate density at which neutrons are absorbed at position $\vec{r}$ due to an isotropic, fixed-source of neutrons, $S(\vec{r})$. In a multiplying system, the production rate density of neutrons due to a fixed source of neutrons, $S(\vec{r})$, can be easily computed from the neutron absorption operator

$$
v \Sigma_{f}(\vec{r}) \int_{4 \pi} \psi(\vec{r}, \hat{\Omega}) d \hat{\Omega}=\frac{v \Sigma_{f}(\vec{r})}{\Sigma_{a}(\vec{r})} \mathbf{A}[S(\vec{r})]
$$

We can now define the right hand side of Eq. (7) as the fission (neutron) production operator, $\mathbf{H}$,

$$
\mathbf{H}[S(\vec{r})] \equiv \frac{v \Sigma_{f}(\vec{r})}{\Sigma_{a}(\vec{r})} \mathbf{A}[S(\vec{r})] .
$$

In the absence of a fixed, external source of neutrons, the neutron source $S(\vec{r})$ within the problem will be solely due to fission events. In this case, the fission source density must satisfy the eigenvalue equation

$$
\mathbf{H}[S(\vec{r})]=\lambda S(\vec{r}) .
$$

Equation (9) gives the traditional eigenvalue formulation of the neutron transport equation for a multiplying (fissionable) material. Unfortunately, relatively little can be said about the general properties of the fission production operator $\mathbf{H}$. Available information about the properties of $\mathbf{H}$ can be found in References 6 and 7. For this paper we will simply assume that $\mathbf{H}$ is a linear operator with (discrete) eigenvalues $\left\{\lambda_{1}, \ldots, \lambda_{\infty}\right\}$ and corresponding eigenfunctions $\left\{b_{1}(\vec{r}), \ldots, b_{\infty}(\vec{r})\right\}$ such that

$$
\mathbf{H}\left[b_{n}(\vec{r})\right]=\lambda_{n} b_{n}(\vec{r}) .
$$

Furthermore, we will assume that the eigenvalues of $\mathbf{H}$ are ordered such that $\left|\lambda_{1}\right|>\left|\lambda_{2}\right| \geq \ldots\left|\lambda_{\infty}\right| \geq 0$, with $\lambda_{1}$ referred to as the fundamental (or dominant) eigenvalue. We also assume that the set of eigenfunctions form a complete, orthogonal eigenbasis, such that any function $f(\vec{r})$ can be written as a series expansion in terms of the eigenfunctions

$$
f(\vec{r})=\sum_{n=1}^{\infty} \alpha_{n} b_{n}(\vec{r}) .
$$

Due to the orthogonal properties of the eigenfunctions, we can express the expansion coefficients $\left\{\alpha_{1}, \ldots, \alpha_{\infty}\right\}$ as moments of the original function

$$
\alpha_{n}=\frac{\int f(\vec{r}) b_{n}(\vec{r}) d \vec{r}}{k_{n}},
$$

where $k_{n}$ is the orthonormalization constant given by

$$
k_{n}=\int b_{n}^{2}(\vec{r}) d \vec{r}=\left\|b_{n}(\vec{r})\right\|_{2} .
$$

\section{Monte Carlo Eigenvalue Solution Methods}

The multiplying neutron transport eigenvalue problem defined in Eq. (9) is very general, and can be solved using either deterministic or stochastic (Monte Carlo) methods. Deterministic solution methods typically discretize the variables (in this case the spatial variable) and then solve the resulting eigenvector problem using traditional linear algebra techniques, such as the power method. The power method 
involves the successive application of the transport operator to "power out" the non-fundamental eigenmodes present in an arbitrary initial source vector. After many repeated applications of the transport operator the resulting source vector will converge to the fundamental eigenvector for the problem.

Monte Carlo methods attempt to mimic the deterministic power method by a technique referred to as source iteration. ${ }^{8)}$ In analog source iteration, a MC simulation is used to transport a batch of neutrons from their initial source location until they are either absorbed or leak out of the problem. At each absorption event, new fission sites are randomly created based on the probability of a fission event occurring at that location. Following a normalization process, these sampled fission sites are then used as the source sites for the next iteration.

By successively repeating this process for many iterations the distribution of fission sites will converge towards the fundamental eigenmode, meaning that the fission sites will appear as though they were sampled from a distribution corresponding to the (normalized) fundamental eigenmode. In this paper we seek to express the MC source iteration method using techniques from spectral analysis, in order to be able to relate this stochastic technique with its deterministic cousin, the power method. We will approach this problem by casting the transport eigenvalue problem as an equivalent random sampling problem, which can be solved by MC simulation. Once the relationship between the transport operator and the MC transport process has been established, we will proceed to show the eigenmode convergence and stochastic noise due to a single MC source iteration.

As an initial step in our derivation, we must first impose a condition of neutron accountability upon our problem. That is, for every neutron introduced into the problem we must identify the exact position where the neutron is absorbed. However, for any finite problem there will be some fraction of initial source neutrons that escape the problem before they are absorbed. In the present formulation, the operator A cannot be used to obtain any information about neutrons which escape the problem. To address this issue we employ a vacuum-equivalent black absorber technique previously described by Lux and Koblinger. ${ }^{8)}$ Let us assume that we surround all escape boundaries of the volume $V$ with a thin layer or a perfectly-absorbing, non-fissionable material. This perfect absorber will absorb all neutrons that would otherwise escape the problem, and, because the absorber is placed beyond the original escape boundary, there will be no effect on the absorption reaction rates within the volume $V$. With this vacuum-equivalent absorber in place we are assured that all neutrons born in the problem will be absorbed either within the original volume $V$ or the added external absorber. Conservation of neutrons then requires

$$
\int_{V^{*}} \mathbf{A}[S(\vec{r})] d \vec{r}=\int_{V} S(\vec{r}) d \vec{r},
$$

where $V^{*}$ is the volume of the problem including the external absorber.

Let us now consider the application of the absorption op- erator to a point source of neutrons located at position $\vec{r}_{s}$. Based on the properties of the underlying Green's function (Eq. (3)) we can immediately show that $\mathbf{A}$ is a positive operator

$$
\mathbf{A}\left[\delta\left(\vec{r}-\vec{r}_{s}\right)\right] \geq 0 \quad \forall \quad \vec{r} \in V^{*}, \vec{r}_{s} \in V,
$$

where $\delta$ is the Dirac delta function. Furthermore, by integrating $\mathbf{A}\left[\delta\left(\vec{r}-\vec{r}_{s}\right)\right]$ over the domain $V^{*}$ and applying Eq. (14) we find that

$$
\int_{V^{*}} \mathbf{A}\left[\delta\left(\vec{r}-\vec{r}_{s}\right)\right] d \vec{r}=\int_{V^{*}} \delta\left(\vec{r}-\vec{r}_{s}\right) d \vec{r}=1 .
$$

Based on the results shown in Eqs. (15) and (16) we see that the application of the absorption operator to a point source of neutrons located at position $\vec{r}_{s}$ can be interpreted as a joint probability density function (pdf),

$$
p\left(\vec{r} \mid \vec{r}_{s}\right) \equiv \mathbf{A}\left[\delta\left(\vec{r}-\vec{r}_{s}\right)\right],
$$

which describes the probability that a neutron born (isotropically) at position $\vec{r}_{s}$ will be absorbed within volume $d \vec{r}$ about position $\vec{r}$. Substituting Eq. (17) into Eq. (8) we immediately find that the fission production response to a point source of neutrons located at position $\vec{r}_{s}$ is given by

$$
\mathbf{H}\left[\delta\left(\vec{r}-\vec{r}_{s}\right)\right]=w(\vec{r}) p\left(\vec{r} \mid \vec{r}_{s}\right),
$$

where the weight factor $w(\vec{r})$ is equal to the ratio of the neutron production to neutron absorption rate at position $\vec{r}$,

$$
w(\vec{r}) \equiv \frac{v \Sigma_{f}(\vec{r})}{\sum_{a}(\vec{r})} .
$$

Let us now return to our interpretation of the absorption response due to a point source as a joint pdf, as shown in Eq. (17). This interpretation suggests that unbiased MC neutron transport algorithms may be defined by a very simple criterion: it must be able to produce random samples $\tilde{r}$ from the distribution $p\left(\vec{r} \mid \vec{r}_{s}\right)$ for any given point source $\vec{r}_{s}$. In effect, a MC transport algorithm can be viewed as simply a method for producing random samples for neutron absorption sites. For every neutron born in the problem, an unbiased analog $\mathrm{MC}$ simulation will produce a single sample from the joint pdf characterizing the absorption distribution due to the initial source point. Under this general definition the details of the MC transport process are not important, as long as the method is able to correctly produce samples from $p\left(\vec{r} \mid \vec{r}_{s}\right)$.

For determining fission sites required for $\mathrm{MC}$ eigenvalue calculations Eq. (18) indicates that the random samples taken from $p\left(\vec{r} \mid \vec{r}_{s}\right)$ should not be equally weighted, but instead should be weighted by the function $w(\vec{r})$, which is the ratio of the fission neutron production rate to the neutron absorption rate at position $\vec{r}$. It follows immediately that the total sample weight of particles started during one source iteration of an eigenvalue calculation will not be the same as the weight of particles in the subsequent generation. Thus, as with deterministic methods, a renormalization step is required if the total weight of neutrons is to be preserved between iterations. The details of this renormalization will 
not be covered in this paper, but typically involve a non-analog technique, such as particle splitting/ rouletting, to ensure that each iteration begins with a fixed number of equally-weighted neutrons.

\section{Monte Carlo Fission Response to a Point Source}

In this section we consider the distribution of fission neutrons $S_{1}(\vec{r})$ produced in response to a single neutron emitted from an isotropic point source located at position $\vec{r}_{s}$, which is defined by $S_{0}(\vec{r})=\delta\left(\vec{r}-\vec{r}_{s}\right)$. We will show that any unbiased $\mathrm{MC}$ algorithm, as defined in the previous section, can be used to produce a set of discrete fission site locations that form an unbiased estimate of the true fission neutron source density, $S_{1}(\vec{r})$.

We begin in traditional fashion by using the completeness property (Eq. (11)) of the eigenbasis of $\mathbf{H}$ to express the initial neutron source, $S_{0}(\vec{r})$, as a series expansion in the eigenfunctions of $\mathbf{H}$,

$$
\delta\left(\vec{r}-\vec{r}_{s}\right)=\sum_{n=1}^{\infty} \alpha_{n}\left(\vec{r}_{s}\right) b_{n}(\vec{r}) .
$$

From Eq. (12) we can immediately show that the expansion coefficients $\left\{\alpha_{1}, \ldots, \alpha_{\infty}\right\}$ are completely determined by the location of the initial point source, $\vec{r}_{s}$, and, from Eq. (12), are given by the simple relationship

$$
\alpha_{n}\left(\vec{r}_{s}\right)=\frac{b_{n}\left(\vec{r}_{s}\right)}{k_{n}} .
$$

Substituting Eq. (21) into Eq. (20) and applying the fission production operator $\mathbf{H}$ to the resulting series expansion for the initial point neutron source $S_{0}(\vec{r})$ yields

$$
S_{1}(\vec{r})=\mathbf{H}\left[S_{0}(\vec{r})\right]=\mathbf{H}\left[\sum_{n=1}^{\infty} \frac{b_{n}\left(\vec{r}_{s}\right)}{k_{n}} b_{n}(\vec{r})\right] .
$$

Applying the linearity and eigenvalue properties of the operator $\mathbf{H}$ gives a new series expansion for the resulting fission source $S_{1}(\vec{r})$

$$
S_{1}(\vec{r})=\sum_{n=1}^{\infty} \frac{\lambda_{n} b_{n}\left(\vec{r}_{s}\right)}{k_{n}} b_{n}(\vec{r})=\sum_{n=1}^{\infty} \gamma_{n}\left(\vec{r}_{s}\right) b_{n}(\vec{r}),
$$

where,

$$
\gamma_{n}\left(\vec{r}_{s}\right) \equiv \frac{\lambda_{n} b_{n}\left(\vec{r}_{s}\right)}{k_{n}} .
$$

Equation (23) gives the true fission neutron production function due to an isotropic point source located at $\vec{r}_{s}$. We note that the application of the fission production operator $\mathbf{H}$ has had a smoothing effect on the neutron source function, acting to turn the original point source into a continuous source of fission neutrons distributed over the volume $V$.

With the true fission production response, $S_{1}(\vec{r})$, in hand, we now turn our attention to the properties of a fission production response estimated from an unbiased MC simulation. Given an unbiased, analog MC algorithm that generates random realizations from the pdf $p\left(\vec{r} \mid \vec{r}_{s}\right)$, we can immediately show that each neutron born in $V$ will produce a realization for the location at which the neutron is absorbed, which we will denote $\tilde{r}$. From Eq. (18) it follows that this realization can be used to produce an estimate for the fission production density function, which is given by

$$
\tilde{S}_{1}(\vec{r})=w(\tilde{r}) \delta(\vec{r}-\tilde{r}) .
$$

Simply stated, the MC estimate for the fission production density function is equal to the realized absorption location for the neutron, weighted by the ratio of the fission production to absorption cross section at that location. This result leads us to a key observation about MC methods and their limitations. Whereas the true absorption distribution due to a point source of neutrons is a continuous function, the MC estimate is a point representation that has effectively been sampled from the true continuous solution. It is this discrete representation of a continuous solution that gives rise to the random noise inherent in $\mathrm{MC}$ calculations.

Acknowledging that the MC estimate $\tilde{S}_{1}(\vec{r})$ is a discrete approximation of the true solution $S_{1}(\vec{r})$, we now seek to demonstrate that $\tilde{S}_{1}(\vec{r})$ is an unbiased estimator for $S_{1}(\vec{r})$. Let us consider the series expansion of $\tilde{S}_{1}(\vec{r})$ in the eigenbasis of $\mathbf{H}$, given by

$$
\tilde{S}_{1}(\vec{r})=\sum_{n=1}^{\infty} \frac{w(\tilde{r}) b_{n}(\tilde{r})}{k_{n}} b_{n}(\vec{r})=\sum_{n=1}^{\infty} \tilde{\gamma}_{n}\left(\vec{r}_{s}\right) b_{n}(\vec{r}),
$$

From this formulation we immediately see that the expansion coefficients, which we have denoted $\tilde{\gamma}_{n}\left(\vec{r}_{s}\right)$, are random variables, due to their dependence on the randomly sampled $\vec{r}$. Taking the expected value of the series expansion in Eq. (26) and applying the linearity of the expectation operator,

$$
\mathrm{E}\left[\tilde{S}_{1}(\vec{r})\right]=\sum_{n=1}^{\infty} \mathrm{E}\left[\tilde{\gamma}_{n}\left(\vec{r}_{s}\right)\right] b_{n}(\vec{r})
$$

we see that the expected value of $\tilde{S}_{1}(\vec{r})$ will be equal to $S_{1}(\vec{r})$ if $\mathrm{E}\left[\tilde{\gamma}_{n}\left(\vec{r}_{s}\right)\right]=\gamma_{n}\left(\vec{r}_{s}\right)$ for all $n$. This provides a solid definition for the conditions under which $\tilde{S}_{1}(\vec{r})$ is an unbiased estimator for $S_{1}(\vec{r})$.

With this definition established, we can immediately prove that $\tilde{S}_{1}(\vec{r})$ is an unbiased estimator for $S_{1}(\vec{r})$ by computing the expected values of the random variables $\tilde{\gamma}_{n}\left(\vec{r}_{s}\right)$. Recalling that the random variable $\tilde{r}$ is sampled from the joint pdf $p\left(\vec{r} \mid \vec{r}_{s}\right)$, it follows directly that

$$
\mathrm{E}\left[\tilde{\gamma}_{n}\left(\vec{r}_{s}\right)\right]=\int_{V} \tilde{\gamma}_{n}\left(\vec{r}_{s}\right) p\left(\vec{r} \mid \vec{r}_{s}\right) d \vec{r}=\int_{V} \frac{w(\vec{r}) b_{n}(\vec{r})}{k_{n}} p\left(\vec{r} \mid \vec{r}_{s}\right) d \vec{r} .
$$

Substituting Eq. (18) into Eq. (28) gives

$$
\mathrm{E}\left[\tilde{\gamma}_{n}\left(\vec{r}_{s}\right)\right]=\frac{1}{k_{n}} \int_{V} \mathbf{H}\left[\delta\left(\vec{r}-\vec{r}_{s}\right)\right] b_{n}(\vec{r}) d \vec{r}=\frac{1}{k_{n}} \int_{V} S_{1}(\vec{r}) b_{n}(\vec{r}) d \vec{r} .
$$

By comparing Eq. (29) with Eq. (12) we immediately recognize that the expected value of $\tilde{\gamma}_{n}\left(\vec{r}_{s}\right)$ is equal to the $n^{\text {th }}$ coefficient for the series expansion of $S_{1}(\vec{r})$ in terms of the eigenfunctions of $\mathbf{H}$. Thus, by the definitions provided in Eqs. (23) and (24) we have shown that

$$
\mathrm{E}\left[\tilde{\gamma}_{n}\left(\vec{r}_{s}\right)\right]=\gamma_{n}\left(\vec{r}_{s}\right),
$$

and $\tilde{S}_{1}(\vec{r})$ is, in fact, an unbiased estimator for $S_{1}(\vec{r})$. 
Having computed the expected value of $\tilde{\gamma}_{n}\left(\vec{r}_{s}\right)$, we can also evaluate the variance of $\tilde{\gamma}_{n}\left(\vec{r}_{s}\right)$, which will provide a measure of how closely the MC estimate $\tilde{S}_{1}(\vec{r})$ will match the true solution $S_{1}(\vec{r})$, on an eigenmode by eigenmode basis. Starting from the definition for variance we have

$$
\sigma_{\tilde{\gamma}_{n}}^{2}\left(\vec{r}_{s}\right)=\mathrm{E}\left[\tilde{\gamma}_{n}^{2}\left(\vec{r}_{s}\right)\right]-\mathrm{E}\left[\tilde{\gamma}_{n}\left(\vec{r}_{s}\right)\right]^{2} .
$$

From the preceding discussion, we immediately see that the second term in Eq. (31) is equal to the square of the expansion coefficient for $S_{1}(\vec{r}), \gamma_{n}^{2}\left(\vec{r}_{s}\right)$. The definition in Eq. (24) can be written as

$$
\mathrm{E}\left[\tilde{\gamma}_{n}\left(\vec{r}_{s}\right)\right]^{2}=\gamma_{n}^{2}\left(\vec{r}_{s}\right)=\left(\frac{\lambda_{n} b_{n}\left(\vec{r}_{s}\right)}{k_{n}}\right)^{2} .
$$

Unfortunately, the expected value of $\tilde{\gamma}_{n}^{2}\left(\vec{r}_{s}\right)$ does not yield such a simple and convenient solution. Returning to the definition of $\tilde{\gamma}_{n}\left(\vec{r}_{s}\right)$ given in Eq. (26), we have

$$
\mathrm{E}\left[\tilde{\gamma}_{n}^{2}\left(\vec{r}_{s}\right)\right]=\int_{V} \frac{w^{2}(\vec{r}) b_{n}^{2}(\vec{r})}{k_{n}^{2}} p\left(\vec{r} \mid \vec{r}_{s}\right) d \vec{r} .
$$

Substituting Eq. (18) into Eq. (33) yields

$$
\mathrm{E}\left[\tilde{\gamma}_{n}^{2}\left(\vec{r}_{s}\right)\right]=\int_{V} \frac{w(\vec{r}) b_{n}^{2}(\vec{r})}{k_{n}^{2}} \mathbf{H}\left[\delta\left(\vec{r} \mid \vec{r}_{s}\right)\right] d \vec{r} .
$$

Finally, by writing $\mathbf{H}\left[\delta\left(\vec{r} \mid \vec{r}_{s}\right)\right]$ as a series expansion in its eigenfunctions (Eq. (23)) we obtain

$$
\mathrm{E}\left[\tilde{\gamma}_{n}^{2}\left(\vec{r}_{s}\right)\right]=\int_{V} \frac{w(\vec{r}) b_{n}^{2}(\vec{r})}{k_{n}^{2}} \sum_{n^{\prime}=1}^{\infty} \gamma_{n^{\prime}}\left(\vec{r}_{s}\right) b_{n^{\prime}}(\vec{r}) d \vec{r}
$$

which can be rewritten as

$$
\mathrm{E}\left[\tilde{\gamma}_{n}^{2}\left(\vec{r}_{s}\right)\right]=\sum_{n^{\prime}=1}^{\infty} \frac{\lambda_{n^{\prime}} b_{n^{\prime}}\left(\vec{r}_{s}\right)}{k_{n^{\prime}}} \int_{V} \frac{w(\vec{r}) b_{n}^{2}(\vec{r})}{k_{n}^{2}} b_{n^{\prime}}(\vec{r}) d \vec{r} .
$$

Substituting Eqs. (32) and (36) into Eq. (31) gives a final expression for the variance of $\tilde{\gamma}_{n}\left(\vec{r}_{s}\right)$,

$$
\sigma_{\tilde{\gamma}_{n}}^{2}\left(\vec{r}_{s}\right)=\sum_{n^{\prime}=1}^{\infty} \frac{\lambda_{n^{\prime}} b_{n^{\prime}}\left(\vec{r}_{s}\right)}{k_{n^{\prime}}} \int_{V} \frac{w(\vec{r}) b_{n}^{2}(\vec{r})}{k_{n}^{2}} b_{n^{\prime}}(\vec{r}) d \vec{r}-\left(\frac{\lambda_{n} b_{n}\left(\vec{r}_{s}\right)}{k_{n}}\right)^{2},
$$

as a function of the location of the initial point source, $\vec{r}_{s}$.

Before proceeding, we wish to point out that the result given in Eq. (37) for the variance of $\tilde{\gamma}_{n}\left(\vec{r}_{s}\right)$ is exact, as there are no assumptions or approximations made in the derivation. However, the formula for computing the variance is of limited use since the eigenfunctions of the operator $\mathbf{H}$ are generally not known. The real value of Eq. (37) lies in the fact that we can use this expression to gain insight into the $\mathrm{MC}$ source convergence process, as discussed in the Sections II. 4 and II. 5. It is interesting to notice that the expression for the variance of the expansion coefficient for a single source eigenmode is closely coupled to all of the other eigenmodes in the problem. This indicates that a perturbation introduced into a single eigenmode has the potential to affect the variance by producing noise in all of the other modes. The cross mode coupling term is largely due to the integral embedded in Eq. (37), which may be interpreted as the projection of the square of the eigenfunction of interest onto all other eigenfunctions. Unfortunately, this integral cannot be easily calculated or even estimated unless the detailed properties of the eigenfunctions are known.

\section{Monte Carlo Fission Response to Multiple Indepen- dent Point Sources}

Until this point we have restricted our analysis to a single, isotropic, point source located at position $\vec{r}_{s}$ within the volume $V$. However, as described in Section II. 2, MC transport methods typically represent the neutron source distribution as multiple independent point sources located throughout the volume. Therefore, let us now consider an initial source distribution, $\quad S_{0, M}(\vec{r}), \quad$ containing $\quad M \quad$ independent, equally-weighted, source points. This initial source can be defined as a summation of delta functions,

$$
S_{0, M}(\vec{r})=\frac{C}{M} \sum_{m=1}^{M} \delta\left(\vec{r}-\vec{r}_{m}\right),
$$

where $C$ is a normalization constant such that

$$
\int_{V} S_{0, M}(\vec{r}) d \vec{r}=C
$$

By applying the same methodology used in the previous section to Eq. (38), we can immediately show that the fission neutron density resulting from this initial source is given by the series expansion

$$
S_{1, M}(\vec{r})=\frac{C}{M} \sum_{m=1}^{M} \mathbf{H}\left[\delta\left(\vec{r}-\vec{r}_{m}\right)\right]=\sum_{n=1}^{\infty} \Gamma_{n, M} b_{n}(\vec{r}),
$$

where the expansion coefficients $\Gamma_{n, M}$ are given by

$$
\Gamma_{n, M} \equiv \frac{C}{M} \sum_{m=1}^{M} \frac{\lambda_{n} b_{n}\left(\vec{r}_{m}\right)}{k_{n}}=\frac{C}{M} \sum_{m=1}^{M} \gamma_{n}\left(\vec{r}_{m}\right) .
$$

Applying an unbiased MC transport algorithm to the initial set of source points, $S_{0 M}(\vec{r})$, will produce a set of $M$ independent neutron absorption locations $\left\{\tilde{r}_{1}, \ldots, \tilde{r}_{M}\right\}$, which have been sampled from the joint probability density functions $\left\{p\left(\vec{r} \mid \vec{r}_{1}\right), \ldots, p\left(\vec{r} \mid \vec{r}_{M}\right)\right\}$. From these $M$ samples, it is possible to create an estimate for the fission neutron density as a weighted sum of the sampled absorption points,

$$
\tilde{S}_{1, M}(\vec{r})=\frac{C}{M} \sum_{m=1}^{M} w(\tilde{r}) \delta(\vec{r}-\tilde{r}) .
$$

Expanding Eq. (42) in the eigenbasis of $\mathbf{H}$ yields,

$$
\tilde{S}_{1, M}(\vec{r})=\sum_{n=1}^{\infty} \tilde{\Gamma}_{n, M} b_{n}(\vec{r})
$$

where the expansion coefficients $\tilde{\Gamma}_{n, M}$ are given by

$$
\tilde{\Gamma}_{n, M} \equiv \frac{C}{M} \sum_{m=1}^{M} \frac{w_{n}\left(\tilde{r}_{m}\right) b_{n}\left(\tilde{r}_{m}\right)}{k_{n}}=\frac{C}{M} \sum_{m=1}^{M} \tilde{\gamma}_{n}\left(\vec{r}_{m}\right) .
$$

Again, we see that the MC estimate for the fission neutron density, $S_{0, M}(\vec{r})$, will be unbiased if $\mathrm{E}\left[\tilde{\Gamma}_{n, M}\right]=\Gamma_{n, M}$ for all $n$. This may be easily demonstrated by taking the expected value of Eq. (44) to show

$$
\mathrm{E}\left[\tilde{\Gamma}_{n, M}\right]=\frac{C}{M} \sum_{m=1}^{M} \mathrm{E}\left[\tilde{\gamma}_{n}\left(\vec{r}_{m}\right)\right],
$$


which, by Eq. (30) and Eq. (41), reduces to

$$
\mathrm{E}\left[\tilde{\Gamma}_{n, M}\right]=\frac{C}{M} \sum_{m=1}^{M} \gamma_{n}\left(\vec{r}_{m}\right)=\Gamma_{n, M}
$$

It is also possible to derive an expression for the variance of $\tilde{\Gamma}_{n, M}$ based on the previously obtained result for the variance of $\tilde{\gamma}_{n}\left(\vec{r}_{m}\right)$. Because $\tilde{\Gamma}_{n, M}$ is a sum of $M$ independent random variables (the $\tilde{\gamma}_{n}\left(\vec{r}_{m}\right)$ ), we can use Bienaymé's formula for computing the variance of a sum of uncorrelated variables to show

$$
\sigma_{\tilde{\Gamma}_{n, M}}^{2}=\frac{C^{2}}{M^{2}} \sum_{m=1}^{M} \sigma_{\tilde{\gamma}_{n}}^{2}\left(\vec{r}_{m}\right) .
$$

Substituting Eq. (37) into Eq. (47) yields the final expression for the variance of the $n$th eigenbasis expansion coefficient for $\tilde{S}_{1, M}(\vec{r})$,

$$
\sigma_{\tilde{\Gamma}_{n, M}}^{2}=\frac{C^{2}}{M^{2}} \sum_{m=1}^{M}\left(\sum_{n^{\prime}=1}^{\infty} \frac{\lambda_{n^{\prime}} b_{n^{\prime}}\left(\vec{r}_{m}\right)}{k_{n^{\prime}}} \int_{V} \frac{w(\vec{r}) b_{n}^{2}(\vec{r})}{k_{n}^{2}} b_{n^{\prime}}(\vec{r}) d \vec{r}-\frac{\lambda_{n}^{2} b_{n}^{2}\left(\vec{r}_{m}\right)}{k_{n}^{2}}\right) .
$$

\section{Characteristics of Random Fluctuations due to Monte Carlo Source Iterations}

Equations (46) and (48) give expressions for the expected mean and variance of the eigenbasis expansion coefficients for the MC estimated fission neutron density resulting from a single application of the fission production operator, $\mathbf{H}$, to an initial source consisting of $M$ independent point sources. During MC eigenvalue calculations the method of source iteration is typically used to estimate the fission source distribution corresponding to the fundamental eigenmode of the operator $\mathbf{H}$. During the source iteration process the $\mathbf{H}$ operator is successively applied to an initial neutron source guess consisting of a set of discrete source points as shown in Eq. (38). For each application of the $\mathbf{H}$ operator the eigenmode expansion coefficients for the fission source distribution, $\Gamma_{n, M}$, will (after appropriate normalization) decrease by the ratio of the eigenvalue for the mode to the fundamental eigenvalue. Because the eigenmodes of $\mathbf{H}$ are assumed to be ordered such that $\left|\lambda_{1}\right|>\left|\lambda_{2}\right| \geq \ldots\left|\lambda_{\infty}\right| \geq 0$, it follows that the higher order modes will decay between source iterations, allowing the method to converge to the fundamental mode solution after many iterations.

However, Eqs. (46) and (48) illustrate that the eigenmode expansion coefficients for the fission source distribution produced by a MC algorithm are random variables. Therefore, each application of the fission production operator, $\mathbf{H}$, will produce random fluctuations in the eigenmode expansion coefficients, in addition to the systematic decay of the higher-order coefficients described above. For a single application of the operator $\mathbf{H}$ we can define this random fluctuation as the difference between the true (expected) fission source distribution and the MC realization of the fission source distribution for a common set of initial point sources, which can be written as

$$
\tilde{e}_{M}(\vec{r})=\tilde{S}_{1, M}(\vec{r})-S_{1, M}(\vec{r}) .
$$

This form of Eq. (49) is more typical to analyses in other work that seek to quantify the bias in the $\mathrm{k}_{\mathrm{eff}}$ estimator. ${ }^{9-10)}$ However, these analyses only examine the noise as a whole, rather than the noise associated with individual eigenmodes.

Substituting Eqs. (40) and (43) into Eq. (49) demonstrates that the fluctuation term $\tilde{e}_{M}$ has an eigenbasis expansion,

$$
\tilde{e}_{M}(\vec{r})=\sum_{n=1}^{\infty} \tilde{\varepsilon}_{n, M} b_{n}(\vec{r})
$$

with expansion coefficients given by

$$
\tilde{\varepsilon}_{n, M}(\vec{r}) \equiv\left(\tilde{\Gamma}_{n, M}-\Gamma_{n, M}\right) .
$$

Taking the expected value of Eq. (51) and applying Eq. (46) we can immediately show that $\tilde{\varepsilon}_{n, M}=0$ for all values of $n$. From this intermediate result it follows directly that the expected value of $\tilde{e}_{M}$ is also zero. By taking the variance of Eq. (51) we can also show that

$$
\sigma_{\tilde{\varepsilon}_{n, M}}^{2}=\sigma_{\tilde{\Gamma}_{n, M}}^{2},
$$

Thus we have shown that the random fluctuations introduced to the fission source density during a single $\mathrm{MC}$ source iteration may be expressed as a zero-mean random fluctuation applied to each of the eigenbasis expansion coefficients for the fission source density. We note that this result supports the noise analysis approach used by Toth in Reference 4, as discussed in the introduction.

In his analysis, Toth made three basic assumptions regarding the characteristics of the random noise applied to each eigenmode of the fission source during a source iteration: 1) that the noise is normally distributed with mean zero, 2 ) the variance of the noise is constant and does not vary between eigenmodes, and 3) the noise affecting each eigenmode is independent. Based on the results presented in this paper it is now possible to assess the reasonability of these three assumptions.

We begin by considering Toth's first assumption that the eigenmode fluctuations were normally distributed with mean zero. We have already demonstrated that the fluctuation for each eigenmode has zero mean and variance given by Eq. (48) Also, because the modal fluctuation terms $\tilde{\varepsilon}_{n, M}$ include summations of the point source coefficient realizations, $\tilde{\gamma}_{n}\left(\vec{r}_{m}\right)$, it seems as though it should be possible to apply the Central Limit Theorem (CLT) to prove that the realizations of $\tilde{\varepsilon}_{n, M}$ are normally distributed.

Unfortunately, the underlying realizations for the neutron absorption locations $\left\{\tilde{r}_{1}, \ldots, \tilde{r}_{M}\right\}$ are not identically distributed, so the CLT cannot be applied in its traditional form. However, under certain conditions it is possible to show that the CLT will hold for sums of random variables that are independent, but not necessarily identically distributed. In particular, the Lindeburg condition (or the more restrictive Lypunov's condition) details the conditions under which sums of independent random variables will be normally distributed. Based on general observations of the properties of $\tilde{\gamma}_{n}\left(\vec{r}_{m}\right)$ and $\sigma_{\tilde{\gamma}_{n}}^{2}$, we conjecture that the necessary conditions are, in fact, met and that $\tilde{\Gamma}_{n, M}$ (and hence $\tilde{\varepsilon}_{n, M}$ ) are normally distributed. Unfortunately, a formal proof of this conjecture has not yet been found, and additional discussion 
on this topic is beyond the scope of this current work.

For the second assumption, Toth proposed that the variance of the noise coefficients, $\tilde{\varepsilon}_{n, M}$, was constant for all eigenmodes. Inspection of Eq. (48) illustrates that this assumption is not rigorously true, as the variance of $\tilde{\Gamma}_{n, M}$ has a clear dependence on the corresponding eigenmode. However, numerical studies have demonstrated that the variance of $\tilde{\varepsilon}_{n, M}$ approaches a constant value as the eigenmode order increases, lending additional support to the empirical observations reported by Toth. This behavior is clearly shown in for the simple case considered in Section III. We conjecture that this convergence of the variance of $\tilde{\varepsilon}_{n, M}$ as $n \rightarrow \infty$ holds for all allowable eigenbasis sets for the fission production operator, $\mathbf{H}$, but, again, a proof of this conjecture has not yet been found.

Finally, in his analysis Toth assumed that the noise affecting each eigenmode was independent of the noise affecting the other eigenmodes. Again, we can quickly see that this assumption is not true because the estimates for $\tilde{\varepsilon}_{n, M}$ (as well as $\tilde{\Gamma}_{n, M}$ and $\tilde{\gamma}_{n}\left(\vec{r}_{m}\right)$ ) are clearly correlated with one another. This correlation stems from the fact that the estimates for each mode $n$ are based on the same set of randomly sampled neutron absorption sites $\left\{\tilde{r}_{1}, \ldots, \tilde{r}_{M}\right\}$. In order to correctly account for the effect of this correlation on the variance of the noise expansion coefficients, $\tilde{\varepsilon}_{n, M}$, or the variance of the noise function, $\tilde{e}_{M}$, it is necessary to know the covariance between all pairs of $\tilde{\varepsilon}_{n, M}$ estimates ${ }^{11)}$. While it is possible to derive an expression for this covariance (using a similar approach as was used to compute the variance of $\tilde{\Gamma}_{n, M}$ and $\tilde{\gamma}_{n}\left(\vec{r}_{m}\right)$ ), the resulting expression is more complicated and does not provide any additional insight into the problem.

However, if the variance on every $\tilde{\Gamma}_{n, M}$ is bounded and the variance converges to a fixed value as $n \rightarrow \infty$ (as postulated above), then it is possible to use the maximum variance over all expansion coefficients as a conservatively high variance estimate for each of the $\tilde{\varepsilon}_{n, M}$. This approach will tend to over-predict the noise introduced during each source iteration. In practice, it appears that Toth applied the limiting (large $n$ ) coefficient variance to all of the expansion coefficients, leading to a conservatively large, but reasonable, approximation for the variance of the $\tilde{\varepsilon}_{n, M}$. Thus, we find that the assumptions made by Toth were reasonable for his application, and are largely supported by his results and this theory.

Table 1 Selected eigenvalues for reference slab problem

\begin{tabular}{cc}
\hline$n$ & $\lambda_{n}$ \\
\hline 1 & 1.0762 \\
\hline 2 & 0.8731 \\
\hline 3 & 0.6641 \\
\hline 4 & 0.4975 \\
\hline 5 & 0.3761 \\
\hline 10 & 0.1240 \\
\hline 20 & 0.0337 \\
\hline 50 & 0.0055 \\
\hline
\end{tabular}

\section{Numerical Results}

The results from Section II were verified using a simple, three-dimensional, mono-energetic, analog Monte Carlo code. Testing was performed on a homogeneous $10 \times 100 \times 100$ mean-free-path $(\mathrm{mfp})$ parallelepiped containing a single material with cross-sections $\Sigma_{\mathrm{t}}=1, \Sigma_{\mathrm{s}}=0.7, \Sigma_{\mathrm{a}}=0.3$, $\Sigma_{\mathrm{f}}=0.15$, and $v \Sigma_{\mathrm{f}}=0.37$, also in units of mfp. Neutron scattering within the cube was assumed to be isotropic. The cube had vacuum boundaries in the $x$ direction and reflecting boundary conditions in the $y$ and $z$ directions, effectively making it a 1-D slab problem.

Analytic diffusion theory was used to approximate the eigenfunctions and eigenvalues of the problem, as it had previously been shown ${ }^{12)}$ to provide acceptably accurate approximations to the dominance ratio (DR) for 1-D slab problems. For a 1-D homogemeous slab, diffusion theory predicts flux eigenfunctions of the form

$$
b_{n}(x)=v \Sigma_{f} \sin \left(\frac{n \pi x}{L^{*}}\right),
$$

with corresponding eigenvalues,

$$
\lambda_{n}=\frac{v \Sigma_{f} / \Sigma_{a}}{1+\frac{D}{\Sigma_{a}}\left(\frac{n \pi}{L^{*}}\right)^{2}},
$$

where $D$ is the diffusion coefficient $\left(D=1 /\left(3 \Sigma_{\mathrm{t}}\right)=1 / 3\right)$ and $L^{*}$ is the length of the slab, including the extrapolation distance. For this problem the extrapolation distance was calculated to be $0.7104 \mathrm{mfp},{ }^{13)}$ for a total effective slab length of $L^{*}=11.4208 \mathrm{mfp}$. Using Eq. (54) the fundamental eigenvalue for the reference problem is calculated to be 1.0762 , with a dominance ratio of 0.8113 . The selected eigenvalues for the reference problem are shown in Table 1.

Equation (37) can be used to predict the variance of the expansion coefficients for the fission density resulting from a point source of neutrons located at a fixed position $\vec{r}_{s}$. Figure 1 shows the variance for several expansion coefficients as a function of the initial point source location. It is interesting to note that the variance appears to converge as $n$ increases. This behavior was also seen in several other configurations that were examined.

The MC code described above was used to verify these theoretical results through a series of controlled experiments. For each experiment, one million independent neutron histories were started isotropically at a fixed point. Each neutron was followed until it was either absorbed in the material or leaked out. For every neutron absorption the MC code scored an estimate for the first five $\tilde{\gamma}_{n}\left(\vec{r}_{s}\right)$ coefficients using the estimator given in Eq. (26),

$$
\tilde{\gamma}_{n}\left(\vec{r}_{s}\right)=\frac{w(\tilde{r}) b_{n}(\tilde{r})}{k_{n}} .
$$

After one million histories, the sample variance of each $\tilde{\gamma}_{n}\left(\vec{r}_{s}\right)$ was computed. This process was repeated for 49 initial source point locations, equally spaced across the $10 \mathrm{mfp}$ slab width. Figure 2 shows the experimental variance results as a function of initial source location for the first 5 eigen- 
Table 2 Expected value of eigenmode variance due to $M$ source points sampled from the fundamental eigenmode (sine) of the reference problem. Expected values shown have been multiplied by the number of sampled source points, $M$.

\begin{tabular}{cc}
\hline$n$ & $M \cdot \mathrm{E}\left[\sigma_{\tilde{\Gamma}_{n, M}}^{2}\right]$ \\
\hline 1 & $2.080 \mathrm{E}-3$ \\
\hline 2 & $8.374 \mathrm{E}-3$ \\
\hline 3 & $1.367 \mathrm{E}-2$ \\
\hline 4 & $1.729 \mathrm{E}-2$ \\
\hline 5 & $1.976 \mathrm{E}-2$ \\
\hline 10 & $2.584 \mathrm{E}-2$ \\
\hline 20 & $2.426 \mathrm{E}-2$ \\
\hline 50 & $2.486 \mathrm{E}-2$ \\
\hline
\end{tabular}

modes, compared with the theoretical results predicted by Eq. (37). The results show good agreement between the predicted and measured variance; the shape of the variance is qualitatively correct. The remaining differences are thought to be due to the use of diffusion theory approximations for the eigenfunctions in the theoretical results.

To mimic the behavior of a $\mathrm{MC}$ eigenvalue calculation, the variance of the expansion coefficients resulting from a set of independent neutron source points was considered. In this test, banks of fission source generation sites were formed by taking a fixed number, $M$, of independent samples from the probability density function

$$
p(x)=\frac{1}{7.1323} \sin \left(\frac{\pi x}{L^{*}}\right),
$$

which corresponds to the approximate (normalized) fundamental eigenfunction for the slab.

Instead of computing the variance directly from the points in the sampled bank (using Eq. (48)) we computed the expected variance due to an initial source distributed according to the pdf described in Eq. (56). Taking the expectation of Eq. (47) yields

$$
\mathrm{E}\left[\sigma_{\tilde{\Gamma}_{n, M}}^{2}\right]=\frac{C^{2}}{M} \int_{V} \sigma_{\tilde{\gamma}_{n}}^{2}\left(\vec{r}_{m}\right) p\left(\vec{r}_{m}\right) d \vec{r}_{m} .
$$

Combining Eqs. (37), (56) and (57) it is possible to predict the expected variance for $\tilde{\Gamma}_{n M}$ due to $M$ samples taken from the pdf $p(x)$ The expected variances for several eigenmodes of our test problem are listed in Table 2. Again, we note that the expected value of the variance tends to converge to a constant for large values of $n$.

To test these predictions, a series of numerical experiments were run with the same MC code used for the previous experiments. For each experiment, $M$ neutrons were started isotropically at initial source locations that were sampled from the pdf $p(x)$. Again, the resulting neutron absorption sites were used to score an estimate of the first five $\tilde{\gamma}_{n}\left(\vec{r}_{s}\right)$ coefficients using the estimator given in Eq. (55).

This process was repeated $N$ times for the same set of starting neutron source locations to obtain an estimate of $\sigma_{\tilde{\Gamma}_{u}}^{2}$. Results for $N=100,1000$, and 10000 independent simulations of fixed source banks containing $M=10,100$, and 1000 sampled initial source points from $p(x)$ are given in Table 3.

The results in Table 3 show an inverse relationship between the variance of the expansion coefficients and the number of initial source points, $M$, as predicted by Eq. (57). The measured coefficient variances are also observed to increase with eigenmode order, approaching a constant value as the order increases. This behavior is consistent with the theoretical results shown in Table 2. In general, the experimental results shown in Table 3 show good agreement with the theoretical values given in Table 2 , providing sup-

\begin{tabular}{|c|c|c|c|c|c|c|c|c|c|c|}
\hline $\begin{array}{c}\text { source points } \\
M=10\end{array}$ & \multicolumn{2}{|c|}{$\tilde{\Gamma}_{1,10}$} & \multicolumn{2}{|c|}{$\tilde{\Gamma}_{2,10}$} & \multicolumn{2}{|c|}{$\tilde{\Gamma}_{3,10}$} & \multicolumn{2}{|c|}{$\tilde{\Gamma}_{4,10}$} & \multicolumn{2}{|c|}{$\tilde{\Gamma}_{5,10}$} \\
\hline$N$ & mean & variance & mean & variance & mean & variance & mean & variance & mean & variance \\
\hline 100 & $1.55 \mathrm{E}-01$ & $2.98 \mathrm{E}-04$ & $6.05 \mathrm{E}-03$ & $1.32 \mathrm{E}-03$ & $-6.43 \mathrm{E}-03$ & $1.48 \mathrm{E}-03$ & $-1.81 \mathrm{E}-03$ & $1.80 \mathrm{E}-03$ & $-1.46 \mathrm{E}-03$ & $1.91 \mathrm{E}-03$ \\
\hline 1000 & $1.58 \mathrm{E}-01$ & $2.35 \mathrm{E}-04$ & 7.69E-03 & $9.48 \mathrm{E}-04$ & $-8.66 \mathrm{E}-03$ & $1.43 \mathrm{E}-03$ & $-2.94 \mathrm{E}-03$ & $1.85 \mathrm{E}-03$ & $3.87 \mathrm{E}-04$ & $1.85 \mathrm{E}-03$ \\
\hline 10000 & $1.57 \mathrm{E}-01$ & $2.29 \mathrm{E}-04$ & $6.86 \mathrm{E}-03$ & $9.23 \mathrm{E}-04$ & $-9.90 \mathrm{E}-03$ & $1.45 \mathrm{E}-03$ & $-1.91 \mathrm{E}-03$ & 1.69E-03 & $1.53 \mathrm{E}-03$ & $1.84 \mathrm{E}-03$ \\
\hline $\begin{array}{c}\text { source points } \\
M=100\end{array}$ & \multicolumn{2}{|c|}{$\tilde{\Gamma}_{1,100}$} & \multicolumn{2}{|c|}{$\tilde{\Gamma}_{2,100}$} & \multicolumn{2}{|c|}{$\tilde{\Gamma}_{3,100}$} & \multicolumn{2}{|c|}{$\tilde{\Gamma}_{4,100}$} & \multicolumn{2}{|c|}{$\tilde{\Gamma}_{5,100}$} \\
\hline$N$ & mean & variance & mean & variance & mean & variance & mean & variance & mean & variance \\
\hline 100 & $1.68 \mathrm{E}-01$ & $2.34 \mathrm{E}-05$ & $2.14 \mathrm{E}-03$ & $9.68 \mathrm{E}-05$ & $-1.41 \mathrm{E}-02$ & $1.55 \mathrm{E}-04$ & $1.04 \mathrm{E}-02$ & $1.65 \mathrm{E}-04$ & $9.76 \mathrm{E}-04$ & $2.11 \mathrm{E}-04$ \\
\hline 1000 & $1.67 \mathrm{E}-01$ & $2.30 \mathrm{E}-05$ & $1.41 \mathrm{E}-03$ & $9.17 \mathrm{E}-05$ & $-1.44 \mathrm{E}-02$ & $1.34 \mathrm{E}-04$ & $9.33 \mathrm{E}-03$ & $1.75 \mathrm{E}-04$ & $4.50 \mathrm{E}-04$ & $1.78 \mathrm{E}-04$ \\
\hline 10000 & $1.67 \mathrm{E}-01$ & $2.25 \mathrm{E}-05$ & $1.31 \mathrm{E}-03$ & $9.49 \mathrm{E}-05$ & $-1.41 \mathrm{E}-02$ & $1.42 \mathrm{E}-04$ & $9.99 \mathrm{E}-03$ & $1.77 \mathrm{E}-04$ & $3.68 \mathrm{E}-04$ & $1.92 \mathrm{E}-04$ \\
\hline $\begin{array}{c}\text { source points } \\
M=1000\end{array}$ & \multicolumn{2}{|c|}{$\tilde{\Gamma}_{1,1000}$} & \multicolumn{2}{|c|}{$\tilde{\Gamma}_{2,1000}$} & \multicolumn{2}{|c|}{$\tilde{\Gamma}_{3,1000}$} & \multicolumn{2}{|c|}{$\tilde{\Gamma}_{4,1000}$} & \multicolumn{2}{|c|}{$\tilde{\Gamma}_{5,1000}$} \\
\hline$N$ & mean & variance & mean & variance & mean & variance & mean & variance & mean & variance \\
\hline 100 & $1.65 \mathrm{E}-01$ & $2.93 \mathrm{E}-06$ & $2.80 \mathrm{E}-03$ & 9.34E-06 & $-4.16 \mathrm{E}-03$ & $1.78 \mathrm{E}-05$ & $-3.77 \mathrm{E}-03$ & $2.11 \mathrm{E}-05$ & $-2.28 \mathrm{E}-03$ & $1.82 \mathrm{E}-05$ \\
\hline 1000 & $1.65 \mathrm{E}-01$ & $2.57 \mathrm{E}-06$ & $2.68 \mathrm{E}-03$ & $9.02 \mathrm{E}-06$ & $-4.11 \mathrm{E}-03$ & $1.54 \mathrm{E}-05$ & $-3.62 \mathrm{E}-03$ & $1.68 \mathrm{E}-05$ & $-2.42 \mathrm{E}-03$ & $2.01 \mathrm{E}-05$ \\
\hline 10000 & $1.65 \mathrm{E}-01$ & $2.34 \mathrm{E}-06$ & $2.74 \mathrm{E}-03$ & $9.31 \mathrm{E}-06$ & $-4.15 \mathrm{E}-03$ & $1.46 \mathrm{E}-05$ & $-3.75 \mathrm{E}-03$ & $1.75 \mathrm{E}-05$ & $-2.49 \mathrm{E}-03$ & $1.94 \mathrm{E}-05$ \\
\hline
\end{tabular}

Table 3 Weighted expansion coefficients for $M=10,100$ and 1000 source points sampled from sine distribution 


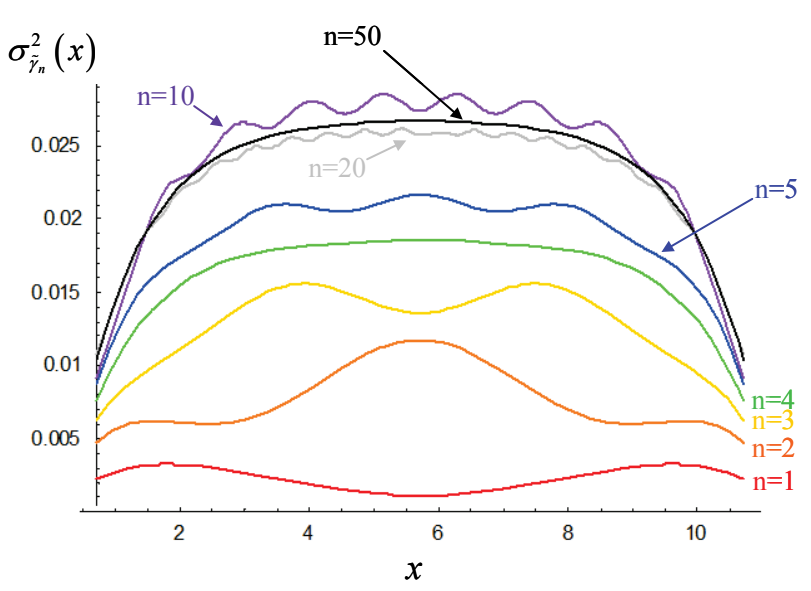

Fig. 1 Predicted variance for several eigenmode expansion coefficients as a function of initial point source location

port for the theory developed in Section II. For the case with $M=1,000$ initial source points and $N=10,000$ independent transport simulations the difference between measured and predicted coefficient variances ranged between $12.5 \%$ (for the first coefficient) and $1.21 \%$ (for the fourth coefficient). Comparison of the measured and predicted data indicates that the theoretical results are biased slightly low; with the theory underpredicting the measured variance in 36 of the 45 test cases considered. We believe that this bias is due to the approximate nature of the eigenfunctions and eigenvalues used to calculate the theoretical results.

\section{Conclusion}

Understanding the propagation of random fluctuations in $\mathrm{MC}$ eigenvalue calculations is important when considering source acceleration schemes or gauging source convergence. Previous research has shown that it is especially important to understand the fluctuations associated with individual eigenmodes. For this reason, we have attempted to provide a more rigorous theoretical foundation for describing the random fluctuations affecting individual eigenmodes by providing a new analysis based on spectral theory. Based on this analysis we make several observations and conjectures regarding the nature of these random fluctuations.

For a fission production operator with a complete set of orthogonal eigenfunctions, we were able to prove that the fluctuations are randomly distributed with mean zero and finite variance. We further conjecture that the noise will be normally distributed in physically realistic cases. Secondly, we conjecture that the variance of the fluctuations are weakly dependent on eigenmode, and will approach a constant value as the eigenmode order increases. While concrete proofs of these conjectures were not reached in this analysis, we did show a strong degree of reasonableness in the assumptions through numerical experiments on a simple 1D problem. With this theoretical foundation laid, further work can be made to make conclusive statements about the propagation of fluctuations in $\mathrm{MC}$ eigenvalue calculations.

\section{Acknowledgment}

The authors would like to thank Dr. Tom Sutton (Knolls

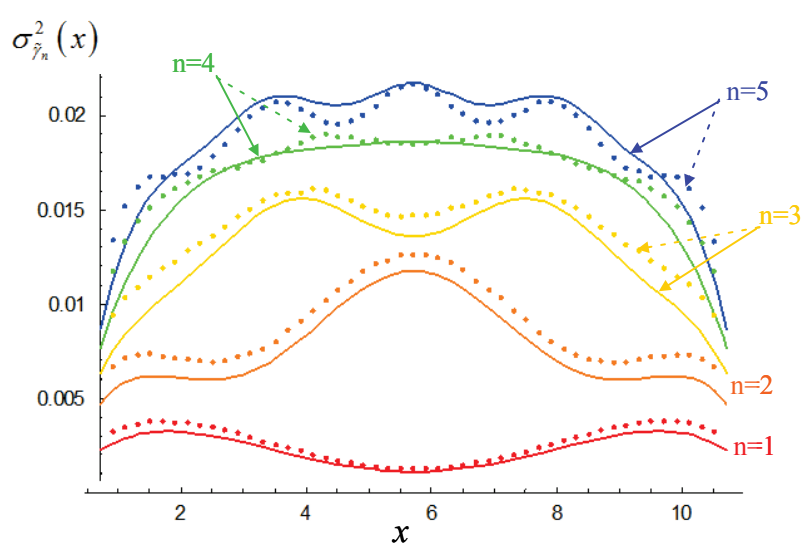

Fig. 2 Predicted (solid line) and measured (points) variance for the first five eigenmode expansion coefficients as a function of initial point source location. Each measurement was obtained from MC simulation of $1 \times 10^{6}$ independent neutron histories started from the corresponding $x$ coordinate.

Atomic Power Laboratory) and Bryan Toth (University of Michigan) for many helpful discussions on Monte Carlo fission source convergence.

\section{References}

1) D. P. Griesheimer, W. R. Martin, J. P. Holloway, "A Functional Expansion Method for Monte Carlo Eigenvalue Calculations,"Proc. of Monte Carlo Method: Versatility Unbounded in a Dynamic Computing World (MC2005), April 17-21, 2005, Chattanooga, USA, (2005), [CD-ROM].

2) D. P. Griesheimer, B. E. Toth, "A Novel Source Convergence Acceleration Scheme for Monte Carlo Criticality Calculations, Part I: Theory," Proc. of Joint Int. Topical Meeting on Mathematics \& Computation and Supercomputing in Nuclear Applications (M\&C+ SNA 2007), April 15-19, 2007, Monterey, USA, (2007), [CD-ROM].

3) B. E. Toth, D. P. Griesheimer, "A Novel Source Convergence Acceleration Scheme for Monte Carlo Criticality Calculations, Part II: Implementation \& Results," Proc. of Joint Int. Topical Meeting on Mathematics \& Computation and Supercomputing in Nuclear Applications (M\&C + SNA 2007), April 15-19, 2007, Monterey, USA, (2007), [CD-ROM].

4) B. E. Toth, W. R. Martin, "Noise Analysis of Smoothed Residual Acceleration, a Monte Carlo Neutron Source Convergence Algorithm," Proc. of Int. Conf. on Mathematics, Computational Methods \& Reactor Phys. (M\&C 2009), May 3-7, 2009, Saratoga Springs, USA, (2009), [CD-ROM].

5) W. Goad, R. Johnson, "A Monte Carlo Method for Criticality Problems," Nucl. Sci. Eng., 5, 371-375 (1959).

6) K. M. Case, P. F. Zweifel, Linear Transport Theory, Addison-Wesley, Reading, Massachusetts (1967).

7) J. J. Duderstadt, W. R. Martin, Transport Theory, Wiley-Interscience, John Wiley \& Sons, New York (1979).

8) I. Lux, L. Koblinger, Monte Carlo Particle Transport Methods: Neutron and Photon Calculations, CRC Press, Ann Arbor, Michigan (1991).

9) B. R. Nease, T. Ueki, T. M. Sutton, F. B. Brown, "Instructive Concepts about the Monte Carlo Fission Source Distribution," Proc. of Int. Conf. on Mathematics, Computational Methods \& Reactor Phys. (M\&C 2009), May 3-7, 2009, Saratoga Springs, USA, (2009), [CD-ROM]. 
10) R. J. Brissenden, A. R. Garlick, "Biases in the Estimation of keff and its Error by Monte Carlo Methods," Ann. Nucl. Energy, 13, 63-83 (1986).

11) D. P. Griesheimer, W. R. Martin, J. P. Holloway, "Convergence Properties of Monte Carlo Functional Expansion Tallies,"J. Comp. Phys., 211, 129-153 (2006).

12) D. K. Parsons, D. E. Kornreich, "Approximations to the Do- minance Ratio using Effective and Infinite Multriplication Results," Proc. of M\&C 2003: A Century in Review - A Century Anew, April 6-11, 2003, Gatlinburg, TN, USA, (2003), [CD-ROM].

13) J. J. Duderstadt, L. J. Hamilton, Nuclear Reactor Analysis, Wiley-Interscience, John Wiley \& Sons, New York (1976). 